\title{
Flora do Espírito Santo: Hypoxidaceae
}

\author{
Flora of Espírito Santo: Hypoxidaceae
}

Julie Henriette Antoinette Dutilh ${ }^{1}$, Edimar Faria Menezes Lopes ${ }^{2}$ \& Antonio Campos-Rocha ${ }^{2,3}$

\begin{abstract}
Resumo
A família Hypoxidaceae é cosmopolita, composta por nove gêneros e aproximadamente duzentas espécies. No Brasil encontra-se representada por três espécies pertencentes aos gêneros Curculigo e Hypoxis. O presente trabalho pretende contribuir para o conhecimento das espécies de Hypoxidaceae ocorrentes no estado do Espírito Santo. Está fundamentado no estudo das coleções depositadas nos herbários visitados, coletas de campo, além da consulta a dados bibliográficos. Para o estado foi registrado somente o gênero Hypoxis, com duas espécies, H. atlantica e H. decumbens. Descrita em 2016 para os estados da Bahia e Santa Catarina, H. atlantica é confirmada para o Espírito Santo. São apresentados descrições das espécies, comentários taxonômicos, dados sobre hábitat, ecologia e distribuição geográfica, além de fotografias. Adicionalmente, é apresentada uma chave de identificação para as espécies.
\end{abstract}

Palavras-chave: Asparagales, Hypoxis, restinga, taxonomia.

\begin{abstract}
Hypoxidaceae has a worldwide distribution, with nine genera and approximately two hundred species. In Brazil, the family is represented by three species, from genera Curculigo and Hypoxis. This work aims to contribute to the knowledge of Hypoxidaceae species in the state of Espirito Santo, Brazil. It is based on the examination of collections deposited in diverse visited herbaria, on fieldwork and bibliography. Only genus Hypoxis was encountered, with two species, H. atlantica and H. decumbens. Described in 2016 for the states of Bahia and Santa Catarina, H. atlantica is confirmed for Espírito Santo. Descriptions of the species, taxonomic, habitat, ecological and distribution data are provided, with photographs. Additionally, an identification key is presented.
\end{abstract}

Key words: Asparagales, Hypoxis, sandy coastal plains, taxonomy.

\section{Introdução}

Hypoxidaceae R.Br. está incluída na ordem Asparagales, a maior entre as monocotiledôneas, juntamente com famílias como Amaryllidaceae, Asparagaceae e Orchidaceae (Chase et al. 2009). Compreende nove gêneros e cerca de duzentas espécies, encontradas nas regiões tropicais e subtropicais de todos os continentes, em sua maioria no hemisfério sul (Nordal 1998; Liu et al. 2012; Govaerts 2017). Na América são registrados dois gêneros, Curculigo Gaertn. e Hypoxis L. (Dutilh 2009), ambos ocorrentes no Brasil (Dutilh 2017). No estado do Espírito Santo a família está representada pelo gênero Hypoxis, com duas espécies.
Este estudo apresenta os resultados do estudo taxonômico das espécies de Hypoxidaceae ocorrentes no estado do Espírito Santo. O tratamento taxonômico constitui-se de descrições morfológicas, comentários taxonômicos e ecológicos, dados sobre distribuição geográfica e hábitat, além de fotografia dos táxons. Em adição, uma chave de identificação para espécies tratadas é fornecida.

\section{Material e Métodos}

Foram visitados os herbários nacionais e internacionais com as coleções mais representativas para o estado do Espírito Santo ou para as Hypoxidaceae brasileiras (BHCB, BM, C, CEPEC,

\footnotetext{
${ }^{1}$ Universidade Estadual de Campinas, Inst. Biologia, Depto. Biologia Vegetal, R. Monteiro Lobato 255, 13083-970, Campinas, SP, Brasil.

${ }^{2}$ Universidade Estadual de Campinas, Inst. Biologia, Depto. Biologia Vegetal, Prog. Pós-graduação em Biologia Vegetal, R. Monteiro Lobato 255, 13083-970, Campinas, SP, Brasil.

${ }^{3}$ Autor para correspondência: camposrocha@hotmail.com
} 
EAC, HRCB, IAC, K, ESA, MBM, MBML, NY, P, RB, SP, SPF, UEC e VIES, siglas segundo Thiers, continuamente atualizado). Bases de dados on-line também foram consultadas $(<\mathrm{http}: / /$ reflora.jbrj.gov.br/>; <http://www.splink.org.br/> e $<$ http://sweetgum.nybg.org/vh $>$ ). Localidades com registros conhecidos foram eventualmente visitadas para obtenção de amostras adicionais, sendo que os espécimes coletados encontramse depositado nos herbários MBML e UEC. A análise morfológica foi realizada com auxílio de microscópio estereoscópico e as medidas obtidas utilizando-se de paquímetro digital. A terminologia adotada para descrição morfológica segue o adotado em Radford et al. (1974), Nordal (1998) e Stearn (2004), com algumas adaptações.

\section{Resultados e Discussão}

No Espírito Santo foram encontradas as duas espécies do gênero Hypoxis com ocorrência mencionada para o Brasil (Funez et al. 2016; Dutilh 2017). Hypoxis atlantica Funez, Hassemer \& J.P.R. Ferreira, descrita ao final de 2016, teve seu registro confirmado para o estado. Hypoxis decumbens L. é uma planta encontrada em diferentes ambientes naturais e, por seu comportamento invasor, comum em ambientes antropizados e urbanos. Apesar disso, revelou-se subcoletado no estado, sendo representando por pouco mais de dez coletas em todo o Espírito Santo.

Hypoxidaceae R.Br., Voy. Terra Austr. 2: 576. 1814. Nom. cons.

Erva com rizoma cormoso vertical, alongado ou globoso, perenes. Folhas radicais, trísticas a rosuladas, pilosas a glabrescentes, margem lisa, reta. Inflorescência com indumento, racemiforme a quase umbeliforme, com 1-várias flores. Flores 3-meras, actinomorfas; sépalas e pétalas em 2 verticilos, hipanto inconspícuo; estames 6, eretos, anteras alongadas com base sagitada ou não; ovário ínfero, 3-locular, óvulos numerosos, anátropos de placentação axilar, estilete filiforme, fistuloso, estigma comissural. Fruto oblongo com indumento; sementes globosas, camada externa de fitomelanina negra.

1. Hypoxis L., Syst. Nat. Ed. 10, 2: 986, 1366. 1759. Erva com rizoma tuberoso globoso, podendo apresentar resquícios de folhas no ápice do rizoma, formando uma túnica apical membranácea ou fibrosa. Raízes fasciculadas, contráteis. Folhas lineares a graminiformes, com bainha glabra formando um pseudocaule mais ou menos evidente acima do rizoma, canaliculadas e carinadas, com indumento mais denso a glabrescentes. Eixo da inflorescência mais curto que as folhas, geralmente piloso (Fig. 1a). Flores longo ou curtamente pediceladas, axilares em brácteas pilosas; sépalas 3 e pétalas 3 , em 2 verticilos, livres entre si ou unidas brevemente na base, sépalas adaxialmente glabras, abaxialmente pilosas a vilosas, pétalas glabrescentes até glabras; estames 6 , com filetes inseridos na base das sépalas e pétalas, anteras basifixas ou dorsifixas, sagitadas; ovário piloso a viloso ou glabrescente. Cápsula com perianto persistente.

Gênero mais representativo da família, possui distribuição semicosmopolita, com cerca de noventa espécies distribuídas pela África, Américas, Ásia e Oceania (Nordal 1998; Singh 2007; Wiland-Szymańska 2009). No Brasil são encontradas duas espécies, $H$. atlantica, restrita a áreas alagadiças de restinga da Bahia à Santa Catarina, e H. decumbens, distribuída por todo o país (Dutilh 2017). No estado do Espírito Santo ambas as espécies foram coletadas.

Nas duas espécies são comuns os indivíduos com flores anômalas, com número variado de sépalas, pétalas ou estames. Algumas destas anomalias foram descritas por Ludwig (1889) para H. decumbens e por Wiland-Szymańska (2002) para uma espécie africana, H. angustifolia Lamarck.

\section{Chave de identificação das espécies de Hypoxidaceae no Espírito Santo}

1. Túnica fibrosa; folhas de $0,8-2,8 \mathrm{~mm}$ larg., carnosas, canaliculadas a carinadas; inflorescência com única bráctea basal; pedicelo maior que bráctea; sementes acastanhadas, opacas, muricadas 1.1. Hypoxis atlantica

1'. Túnica membranácea; folhas de 4-12 mm larg., membranáceas, planas; inflorescência com par de brácteas basais; pedicelo menor que bráctea; sementes negras, lustrosas, com tuberosidades arredondadas ...... 
1.1. Hypoxis atlantica Funez, Hassemer \& J.P.R. Ferreira, Phytotaxa 282: 130, figs. $1 \&$ 5a. 2016.

Fig. 1a-d

Rizoma tuberoso globoso, $0,6-2 \times 0,4-1,8$ $\mathrm{cm}$. Folhas $4-15(-30) \times 0,8-2,8 \mathrm{~mm}$, eretas, carnosas, canaliculadas a carinadas, esparsamente pilosas a glabrescentes, nervura central e laterais mais proeminentes abaxialmente, tricomas quase sempre na região abaxial, nas margens e nervura central, bainhas formando pseudocaule curto ou alongado, circundado pelas fibras remanescentes das bainhas secas. Inflorescência 1-(2-3)-flora. Escapo floral 1-9 cm, filiforme, piloso, brácteas espatais $0,3-7 \times 0,1-0,3 \mathrm{~mm}$, glabras ou pilosas, geralmente 1 na base da flor basal da inflorescência e 1 na base de cada uma das demais flores, com tufo apical de tricomas. Flores com pedicelo 4-20 mm, amarelas, sépalas 4-6 × 1,5-2 mm, adaxialmente glabras, abaxialmente pilososas, pétalas podendo ser pouco menores, glabras, filetes 1-2 mm, anteras $1-1,5 \mathrm{~mm}$, base sagitada, ovário $0,8-1 \mathrm{~cm}$ comp, elíptico, estilete $3 \mathrm{~mm}$, estigma curtamente trífido comissural. Fruto cápsula 1-1,3 cm comp., esparsamente piloso, deiscência circunséssil geralmente com permanência da parte apical parcialmente aderente e depois loculicida, permanecendo ereto. Sementes $0,8-1,1 \mathrm{~mm}$ comp., largamente elípticas, duras, acastanhadas, opacas, muricadas, cobertas por projeções persistentes piramidais com aparência de película.

Material examinado: Guarapari, Parque Estadual Paulo Cesar Vinha, 20,5974 ${ }^{\circ} \mathrm{S}, 40,4115^{\circ} \mathrm{W}, 25 . \mathrm{XI} .2006$, fl., R.T. Valadares 352 (VIES); 3.X.2016, fl. e fr., A. CamposRocha et al. 1623 (MBML, UEC); 13.XI.2016, fl. e fr., A. Campos-Rocha \& W.S. Borges 1646 (MBML, UEC). Vitória, Reserva Ecológica de Camburi, 20¹5'60"S, 40¹5'59”'W, 2.IX.1998, fl. e fr., A.M. Assis 596 (VIES).

Espécie descrita recentemente a partir de poucos materiais coletados nos estados da Bahia e Santa Catarina, em solos arenosos de locais alagadiços da região litorânea. Funez et al. (2016) consideraram-na uma espécie rara e deficiente de dados, com ocorrência confirmada apenas para os estados da Bahia e Santa Catarina. Posteriormente, foram identificadas coletas realizadas nos estados
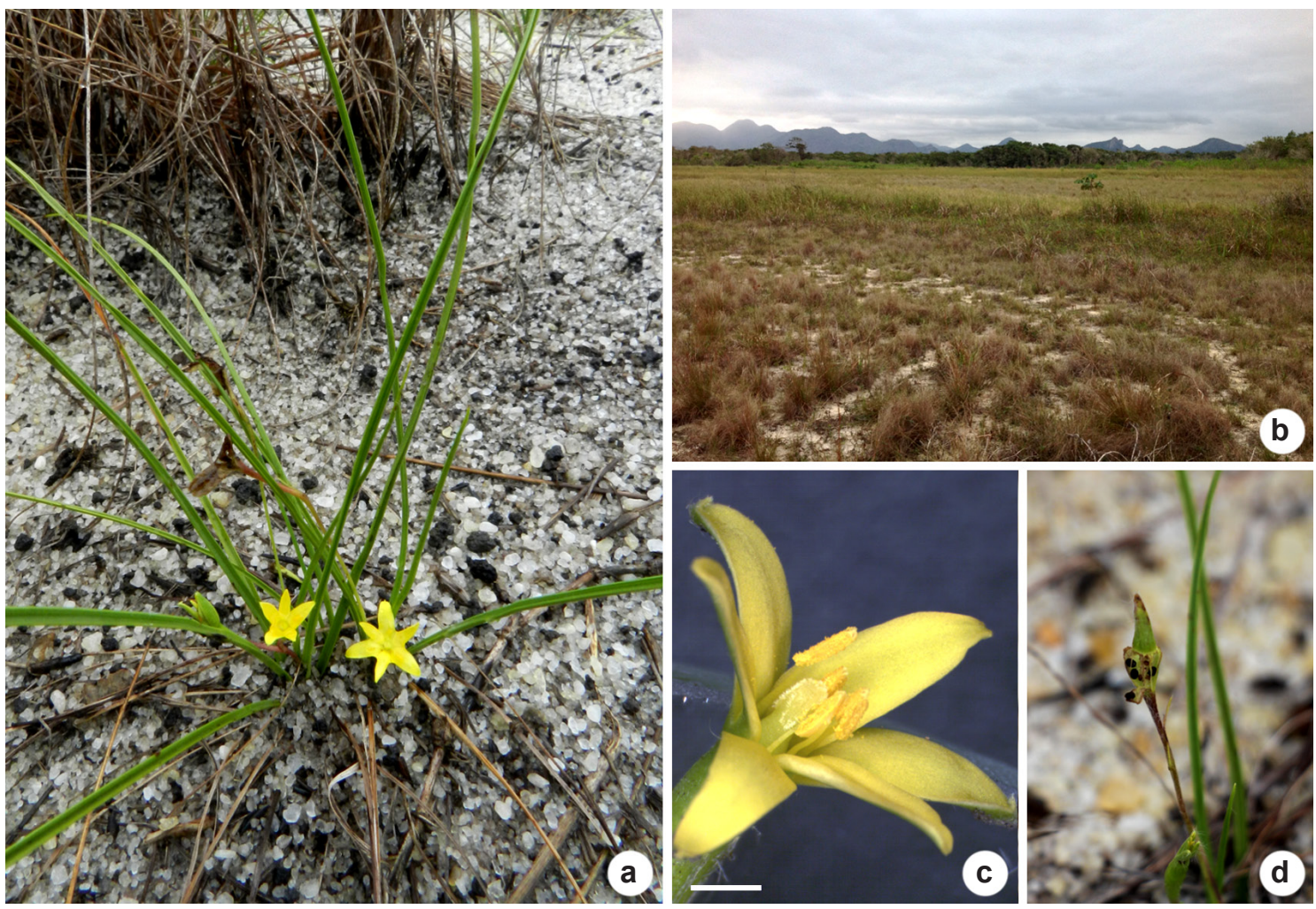

Figura 1 - a-d. Hypoxis atlantica - a. hábito; b. hábitat (Parque Estadual Paulo Cesar Vinha, Guarapari - ES); c. detalhe da flor; $d$. fruto maduro expondo as sementes. (c. barra de escala $=1 \mathrm{~mm}$ ).

Figure 1 - a-d. Hypoxis atlantica - a. habit; b. habitat (Parque Estadual Paulo Cesar Vinha, Guarapari - ES); c. detail of flower; d. mature capsule exposing the seeds. (c. scale bar $=1 \mathrm{~mm}$ ). 
do Rio de Janeiro e Espírito Santo. Neste, foi coletada somente em unidades de conservação da região metropolitana de Vitória. Caracterizase por apresentar rizoma cormoso de túnicas fibrosas, folhas estreitas carnosas e mais eretas, inflorescência uniflora ou biflora, raro triflora, além de sementes amarronzadas e muricadas. Assemelha-se principalmente com $H$. wrightii (Baker) Brackett, descrito a partir de materiais de Cuba, que apresentam o mesmo tipo de folhas, inflorescência e sementes (Herndon 1988, 1992).
1.2. Hypoxis decumbens L., Sp. Pl.: 473. 1753.

Fig. 2a-d

Rizoma tuberoso globoso, 0,6-2 × 0,5-1,8 $\mathrm{cm}$. Folhas 9-45 × 0,4-1,2 cm, ápice acuminado, pilosas a glabrescentes, às vezes com tricomas somente em algumas áreas da folha, como margens e nervuras, tricomas pedunculados, com 1-2(-5) ramos, cada ramo podendo medir até $0,5-2$ a mais de 3,5 mm de comprimento; nervura central conspícua convexa abaxialmente, geralmente duas nervuras laterais maiores convexas adaxialmente,
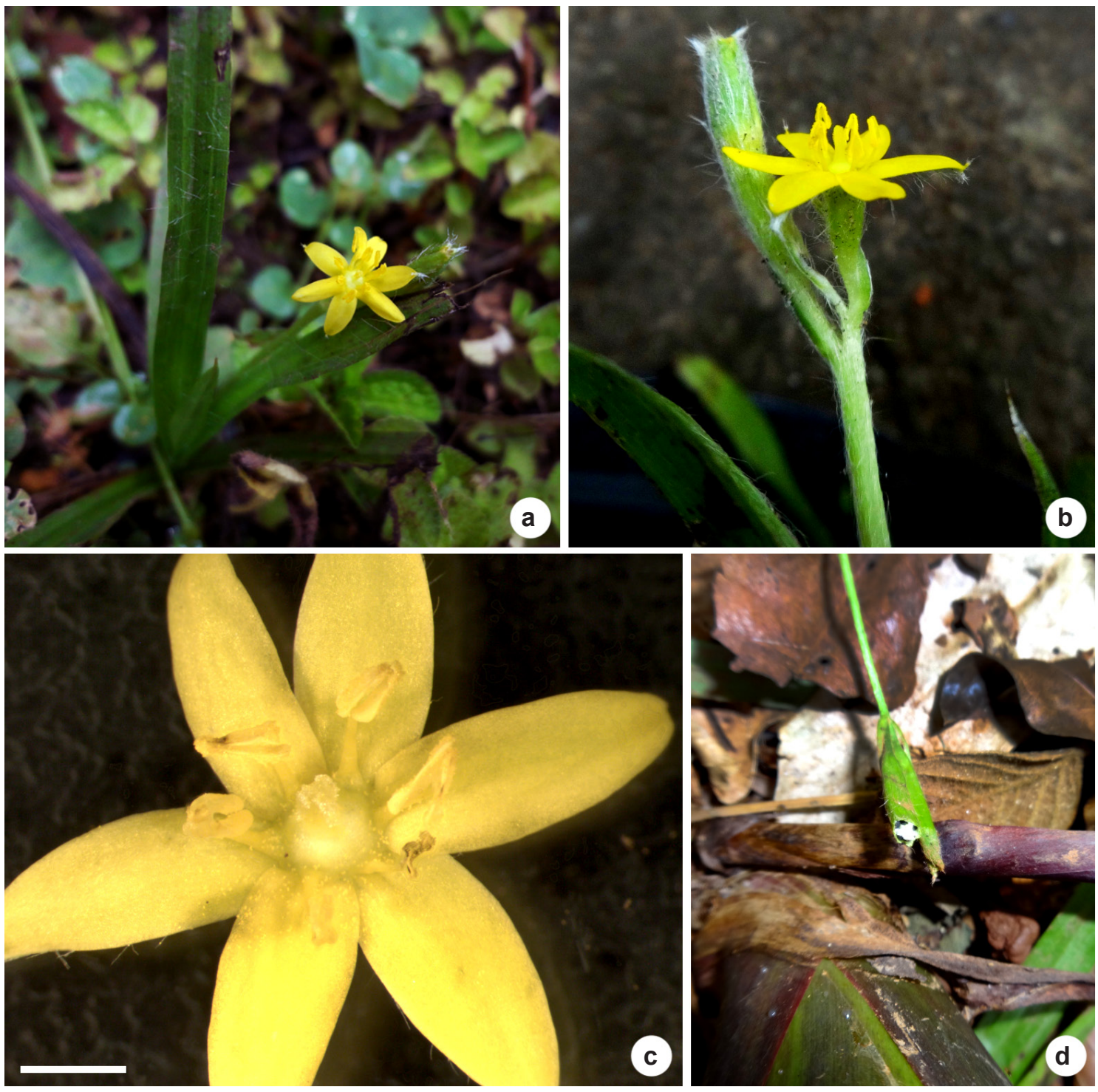

Figura 2 - a-d. Hypoxis decumbens - a. hábito; b. detalhe da inflorescência; c. detalhe da flor, visão superior; d. fruto maduro expondo as sementes. (c. barra de escala $=1 \mathrm{~mm})$.

Figure 2 - a-d. Hypoxis decumbens - a. habit; b. detail of inflorescence; c. detail of flower, upper view; d. mature capsule exposing the seeds. (c. scale bar $=1 \mathrm{~mm})$. 
com a folha apresentando uma forma plissada em corte transversal, bainhas formando pseudocaule curto ou alongado, circundado pelos remanescentes membranáceos das bainhas secas. Escapo floral 1,5-25 cm compr., axilar, geralmente ancipitado na base, piloso, com tricomas geralmente com 2 ramos, brácteas espatais $1-18 \mathrm{~mm}$ compr., lineares, geralmente 2 na base das 2 flores basais da inflorescência e 1 na base de cada uma das demais flores, pilosas, com tricomas com 2-3 ramos. Inflorescência 1-6 flora, racemosa a umbeliforme. Flores com pedicelo ca. 1-10 mm compr., amarelas; sépalas 4-8 × 1-2,5 mm, abaxialmente esverdeadas e muito pilosas a lanosas, com tricomas com 2-mais ramos, adaxialmente amarelas e com tufo de papilas esbranquiçadas no ápice; pétalas pouco mais curtas e mais estreitas, amarelas, glabras ou com faixa central longitudinal abaxial de tricomas; filetes subulados 2-4 mm compr., iguais a subiguais, frequentemente 1-2 mais longos, anteras 1-2 mm comp., bitecas, presas no filete pela base do conectivo, com uma curvatura e espessura reduzida no ápice do filete, como se fosse dorsifixa, versátil, base da antera geralmente sagitada, ápice geralmente mais unido, com deiscência longitudinal lateral; ovário 3-8 $\mathrm{mm}$ compr., elíptico a clavado estilete $2-4 \mathrm{~mm}$ compr., curtamente trífido mas com os ápices permanecendo unidos simulando um estilete único e área estigmática nas bordas dos lobos unidos, formando um estigma do tipo comissural. Fruto cápsula 4-9 mm compr., decumbente a prostrado, deiscência circunséssil irregular, com perda do ápice e frequentemente loculicida; sementes 0,5-1 mm compr., subglobosas, duras, negras, lustrosas, ápice mucronado, com tuberosidades obtusas a fracamente afiladas mais ou menos densamente agrupadas na superfície.

Material examinado: Alegre, Parque Estadual da Cachoeira da Fumaça, trilha da Maria Luzia Campagnaro, 649 m, 20³7'28”S, 41³6'26”W, 11.VI.2010, fl., M. Milward de Azevedo et al. 279 (RB, VIES); trilha do Sr. Jaci, 449 m, 20³7'51'S, 41³6'15'W, 11.VI.2010, fl., M. Milward de Azevedo et al. 257 (VIES); Parque Nacional do Caparaó, Pedra Roxa, Margem do Rio Pedra Roxa, 18.X.2000, fl., W. Foster \& G.O. Romão (ESA). Castelo, Parque Estadual do Forno Grande, Mirante, 20³1'10"S, 41 '5'15'W, 7.IV.2009, fl. e fr., R.C. Forzza et al. 5481 (CEPEC, RB). Guarapari, Parque Natural Municipal Morro da Pescaria, 8.IX.2013, A.C.S Dal Col \& J. Rodrigues-Filho 138 (VIES). Ibitirama, Santa Marta, Parque Nacional do Caparaó. Vale do Rio Santa Marta, 10.IV.2012, H.M. Dias et al. 677 (VIES). Iúna, comunidade do Rio Claro, Albergue do Cedro, próximo ao rio, 1079 m, 19.III.2014, fl. e fr., M. Monge et al. 2719 (UEC). Santa Leopoldina, Chaves, 20³'18”S,
40³2’20”W, 17.I.2008, fl. e fr., L.A. Pereira et al. 1565 (RB). Santa Maria de Jetibá, Garrafão, Sítio Renascer, 208'60'S, 4049'0"'W, 4.VII.2009, T.S. Lorencini 305 (VIES). Santa Teresa, 27.XII.1985, G.F. Santos (VIES951). Serra, APA Mestre Álvaro, 10.III.2012, P.H.D. Barros et al. 158 (VIES). Vitória, Campus da UFES, 26.X.1987, O.J. Pereira et al. 1221 (VIES).

Espécie encontrada com frequência em áreas mais abertas, úmidas, próximas a matas, ou antropizadas de todo o Brasil, onde se destacam pelas pequenas flores amarelas abertas durante o dia. Distribui-se do México a Argentina, sendo registrada na maior parte do Brasil, sobretudo em sua porção leste. No Espírito Santo foi coletada em diferentes ambientes, principalmente em unidades de conservação, apesar de ser uma espécie muito comum em ambientes antrópicos.

\section{Agradecimentos}

Aos curadores e equipe dos herbários consultados; ao Programa de Pós-Graduação em Biologia Planta/IB-UNICAMP, à CAPES e ao CNPq, as bolsas de Doutorado concedidas a A.C.R. e E.F.M.L.; ao Instituto Estadual do Meio Ambiente do Espírito Santo (IEMA), a autorização de nossas pesquisas; a Danilo A.V. Lima, a autorização para a reprodução da imagem dos frutos de $H$. atlantica (Fig. 1d); aos revisores, seus valiosos comentários e críticas.

\section{Referências}

Chase MW, Reveal JW \& Fay MF (2009) A subfamilial classification for the expanded asparagelean families Amaryllidaceae, Asparagaceae and Xanthorrhoeaceae. Botanical Journal of the Linnean Society 161: 132-136.

Dutilh JHA (2009) Neotropical Hypoxidaceae. In: Milliken W, Klitgård B \& Baracat A(2009 onwards). Neotropikey - interactive key and information resources for flowering plants of the Neotropics. Disponível em <http://www.kew.org/science/ tropamerica/neotropikey/families/Hypoxidaceae. htm>. Acesso em 13 abril 2017.

Dutilh JHA (2017) Hypoxidaceae. In: Flora do Brasil (2020 em construção) Jardim Botânico do Rio de Janeiro. Disponível em <http://floradobrasil.jbrj. gov.br/reflora/floradobrasil/FB134>. Acesso em 24 maio 2017.

Funez LA, Hassemer G \& Ferreira JPR (2016) Hypoxis atlantica (Hypoxidaceae): a rare new species endemic to coastal eastern Brazil. Phytotaxa 282: 129-138.

Govaerts R (2017) World checklist of Hypoxidaceae. Facilitated by the Royal Botanic Gardens, Kew. Disponível em $<$ http://apps.kew.org/wcsp/>. Acesso em 29 maio 2017. 
Herndon A (1988) Ecology and systematics of Hypoxis sessilis and H. wr ightii (Hypoxidaceae) in Southern Florida. American Journal of Botany 75: 1803-1812.

Herndon A (1992) The genus Hypoxis (Hypoxidaceae) in Florida. Florida Scientist 55: 45-55.

Liu KW, Xie GC, Chen LJ, Xiao XJ, Zheng YY, Cai J, Zhai JJ, Zhang GQ \& Liu ZJ (2012) Sinocurculigo, a new genus of Hypoxidaceae from China based on molecular and morphological evidence. PLoS ONE 7: e38880.

Ludwig F (1889) Beobachtungen von Fritz Müller an Hypoxis decumbens. Flora oder Botanische Zeitung 72: $55-56$.

Nordal I (1998) Hypoxidaceae. In: Kubitzki K (ed.) The families and genera of vascular plants. Vol. 3. Springer, Berlin. Pp. 286-295.

Radford AE, Dickison WC, Massey JR \& Bell CR (1974) Vascular plant systematics. Harper \& Row, New York. 891p.
Singh Y (2007) Hypoxis (Hypoxidaceae) in southern Africa: taxonomic notes. South African Journal of Botany 77: 360-365.

Stearn WT (2004) Botanical latin. 4ª ed. Timber Press, Portland. 560p.

Thiers B [continuamente atualizado]. Index herbariorum: a global directory of public herbaria and associated staff. New York Botanical Garden's Virtual Herbarium. Disponível em <http:// sweetgum.nybg.org/science/ih/>. Acesso em 25 maio 2017.

Wiland-Szymańska J (2002) Taxonomic and morphological notes on Hypoxis angustifolia (Hypoxidaceae) from Africa, Madagascar, and Mauritius. Novon 12: 142-151.

Wiland-Szymańska J (2009) The genus Hypoxis L. (Hypoxidaceae) in the East Tropical. Biodiversity Research and Conservation 14: 1-129. 